\title{
Quasinormal modes for the scattering on a naked Reissner-Nordström singularity
}

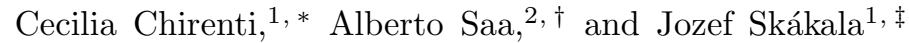 \\ ${ }^{1}$ Centro de Matemática, Computação e Cognição, UFABC, 09210-170 Santo André, SP, Brazil \\ ${ }^{2}$ Departamento de Matemática Aplicada, Universidade Estadual de Campinas, 13083-859 Campinas, SP, Brazil
}

\begin{abstract}
What should be the quasinormal modes associated with a spacetime that contains a naked singularity instead of a black hole? In the present work we address this problem by studying the scattering of scalar fields on a curved background described by a Reissner-Nordström spacetime with $|q|>m$. We show that there is a qualitative difference between cases with $1<q^{2} / m^{2} \lesssim 9 / 8$ and cases with $q^{2} / m^{2} \gtrsim 9 / 8$. We discuss the necessary conditions for the well-posedness of the problem, and present results for the low damped modes in the low $l$ and large $l$ limit. We also consider the asymptotically highly damped quasinormal modes. We present strong evidence that such modes are absent in the case of a naked Reissner-Nordström singularity, corroborating recent conjectures relating them to classical and quantum properties of horizons.
\end{abstract}

PACS numbers: 04.25.dc, 04.30.Nk, 04.70.Bw

\section{INTRODUCTION}

The naked Reissner-Nordström (R-N) singularity is a classical general relativistic solution in electrovacuum. The solution is expected to have a very limited meaning, due to the fact that such singularities cannot be created neither by a gravitational collapse, nor by dropping a charge into the black hole. (According to the weak cosmic censorship conjecture general naked singularities should be prohibited in general theory of relativity, although there are indications that by including quantum effects the violations of the conjecture could be considered [1].) Moreover, a naked singularity created from some exotic initial data conditions should become quickly neutralized (classically, or via quantum pair production). Some results also indicate that if one considers electrogravitational perturbations the R-N naked singularity becomes linearly unstable [2]. However it was discovered that the scalar field scattering problem on such a singular background can be still well defined [3 8$]$, since the waves remain regular at the origin. (However, the back reaction of the given scalar field configuration might still excite some of the unstable electro-gravitational modes and this would eventually lead to a breakdown of the perturbation approach.) Despite the nice regularity property of the scattering problem, the spacetime is non-globally hyperbolic and the time evolution of the fields is not unique [9, 10]. This means one has to specify an additional boundary condition at the singularity to obtain a fully unique time evolution. Another way of seeing the problem is through the language of operators: one can understand the spatial part of the wave operator as a positive symmetric operator acting on a $L^{2}$ Hilbert space, and then obtain the scalar field dynamics through a suitable positive self-adjoint extension of such a symmetric

\footnotetext{
* e-mail: cecilia.chirenti@ufabc.edu.br

$\dagger$ e-mail: asaa@ime.unicamp.br

$\ddagger$ email: jozef.skakala@ufabc.edu.br
}

operator [3, 4]. (One "preferred" way in which such a self-adjoint extension can be always realized is through the so called Friedrich's extension [3], which will also be the case of this paper.) Anyway, after uniquely specifying the dynamics, one should be able to characterize the scattering by a set of characteristic oscillations, the quasi-normal modes.

Low damped quasi-normal modes are in general used as a possible source of information about potential astrophysical objects (such as neutron stars, black holes), and the highly damped modes are potentially interesting from the point of view of quantum gravity [11, 12] 1] Since a lot of work was devoted to the problem of quasinormal modes of the Reissner-Nordström black hole, it might be interesting to observe what happens if one transits from the R-N black hole case to the R-N naked singularity case (with a reflective boundary condition). Information about "what happens" shows how many features of the quasinormal modes of the black hole spacetimes are specific to the black holes themselves and what features survive much more general conditions. Let us also give one concrete example why asking what happens with the quasinormal modes of the naked R-N singularity might be interesting: In the black hole case the behavior of the asymptotically highly damped modes is widely suspected to be linked to the properties of the black hole spacetime horizon(s), (or more specifically it is considered to carry information about quantum black holes). What does then happen with the asymptotically highly damped modes in case there are no horizons present? (If the behavior of the highly damped modes in case of R-N naked singularity would, for instance, resemble the behavior of the highly damped modes of the black hole, it would be a disturbing fact from the point of view of the popular conjectures linking the modes to the horizon's quantum area spacing. Moreover, investigating the R-N

\footnotetext{
${ }^{1}$ For a paper dealing particularly with the R-N black hole case see [13].
} 
naked singularity from this point of view is especially attractive, as the $\mathrm{R}-\mathrm{N}$ naked singularity is obtained by a continuous transition in the $q, m$ parameters from the R-N black hole spacetime.) Thus, briefly, we hope that despite the fact that most likely the R-N naked singularity model does not correspond to a realistic physical situation, there are still many interesting things one can learn from such a model.

The structure of this paper is as follows: In the second section we analyse the problem of the uniqueness of the time evolution of scalar fields on a R-N naked singularity background. In the sections three and four we analyse the properties of the effective potential for the scalar fields scattering and the geometrical optics (eikonal) limit of such a scattering problem. In the fifth section we define analytically solvable potentials that can give good approximations to the problem of the low damped QNM frequencies (such that characterize the given scattering problem). In the sixth section we use those analytical approximations to derive semi-analytical results for the QNM frequencies in the eikonal limit. In the seventh section we use the numerical characteristic integration to obtain the low damped frequencies for the low values of l. In the eighth section we analyse what happens with the asymptotically highly damped modes and we suggest that in case of naked R-N singularity such modes do not exist (as one might expect considering some presently popular conjectures [11, 12]). We give the final conclusions in the section nine. We provide also appendices with more detailed results and some further suggestions for the analytical approximations of the problem.

\section{THE TIME EVOLUTION PROBLEM FOR A SCALAR FIELD IN THE R-N NAKED SINGULARITY}

In this section we will follow the standard analysis of the scalar field evolution in a curved background. (As an example of such an analysis see the treatment of Schwarzschild black hole perturbations in [14, 15]. For a review that presents also such techniques see for example [16].) Take the Klein-Gordon equation for the complex (charged) scalar field:

$$
\frac{1}{\sqrt{-g}} \partial_{\mu}\left(\sqrt{-g} g^{\mu \nu} \partial_{\nu} \Psi\right)=0
$$

with the metric line element given as

$$
g_{\mu \nu} d x^{\mu} d x^{\nu}=-f(r) d t^{2}+f(r)^{-1} d r^{2}+r^{2} d \Omega^{2} .
$$

For the Reissner-Nordström (R-N) singularity the function $f(r)$ is in Planck units given as:

$$
f(r)=1-\frac{2 m}{r}+\frac{q^{2}}{r^{2}}, \quad\left(q^{2}>m^{2}\right) .
$$

Take the decomposition of the field into the spherical harmonics

$$
\Psi(t, r, \theta, \phi)=\sum_{l, m} \psi_{l}(t, r) Y_{m l}(\theta, \phi) .
$$

After we separate the variables we obtain the following reduced equation

$$
\begin{aligned}
\frac{d^{2} \psi_{l}(t, r)}{d t^{2}} & =\frac{f(r)}{r^{2}} \frac{d}{d r}\left[r^{2} f(r) \frac{d \psi_{l}(t, r)}{d r}\right] \\
& -\frac{l(l+1) f(r)}{r^{2}} \psi_{l}(t, r) .
\end{aligned}
$$

We are interested only in compactly supported data initial value problem: first assume that $\psi_{l}(r, t)$ is everywhere bounded and hence the Laplace transform of $\psi_{l}(r, t)$ exists:

$$
\tilde{\psi}_{l}(s, r)=\int_{0}^{\infty} d t e^{-s t} \psi_{l}(t, r)
$$

The Laplace transformed equation (5) gives the following equation:

$$
\begin{aligned}
s^{2} \tilde{\psi}_{l}(s, r) & =\frac{f(r)}{r^{2}} \frac{d}{d r}\left[r^{2} f(r) \frac{d \tilde{\psi}_{l}(s, r)}{d r}\right] \\
& -\frac{l(l+1) f(r)}{r^{2}} \tilde{\psi}_{l}(s, r)+I_{l}(s, r),
\end{aligned}
$$

where

$$
I_{l}(s, r)=\left[s \psi_{l}(t, r)+\frac{d \psi_{l}(t, r)}{d t}\right]_{\mid t=0} .
$$

The solution that corresponds to the initial data term $I_{l}(s, r)$ is obtained by the inverse Laplace transform of $\tilde{\psi}_{l}(s, r)$ and the function $\tilde{\psi}_{l}(s, r)$ is given as

$$
\tilde{\psi}_{l}(s, r)=\int_{0}^{\infty} d r^{\prime} G_{l}\left(s, r, r^{\prime}\right) I_{l}\left(s, r^{\prime}\right) .
$$

Here $G_{l}\left(s, r, r^{\prime}\right)$ is a Green's function satisfying

$$
\begin{aligned}
& -\frac{f(r)}{r^{2}} \frac{d}{d r}\left[r^{2} f(r) \frac{d G_{l}\left(s, r, r^{\prime}\right)}{d r}\right] \\
& +\left[s^{2}+\frac{l(l+1) f(r)}{r^{2}}\right] G_{l}\left(s, r, r^{\prime}\right)=\delta\left(r-r^{\prime}\right) .
\end{aligned}
$$

Since $\psi_{l}(r, t)$ was bounded, its Laplace transform $\tilde{\psi}_{l}(s, r)$ must be also bounded in $r$. This translates to the boundedness of the Green's function in $r$. The unique solution of the given initial data problem is obtained if the condition of boundedness of the Green's function leads to a unique way to construct Green's function from the two linearly independent solutions $U_{l 1}, U_{l 2}$ of the homogeneous equation

$$
\begin{aligned}
& -\frac{f(r)}{r^{2}} \frac{d}{d r}\left[r^{2} f(r) \frac{d U_{l 1,2}(s, r)}{d r}\right] \\
& +\left[s^{2}+\frac{l(l+1) f(r)}{r^{2}}\right] U_{l 1,2}(s, r)=0 .
\end{aligned}
$$


If $f(r)$ goes to 1 at spatial infinity (the metric is asymptotically flat), then there is only one solution of (11) that stays bounded as $r \rightarrow \infty$. If there exists only one solution of the equation (11), such that it is linearly independent from the solution bounded at infinity and in the same time it is bounded at $r \rightarrow 0$ then these two solutions uniquely define the Green's function. If all the solutions of (11) are singular at 0 , there is no solution (bounded in $r$ ) of the given initial value problem such that it can be Laplace transformed (this can be taken as an indication that there is no solution at all).

If both of the linearly independent solutions are regular at 0 , and (at least) two different Green functions lead to a function in the domain of the inverse Laplace transform, then there is no uniquely defined solution to the initial value problem. In the "worst" case there are infinitely many solutions, given by arbitrary linear combination of $U_{l 1}, U_{l 2}$, that are linearly independent to the solution bounded at $r \rightarrow \infty$. In such a case the problem is underdetermined and one needs one more condition at $r=0$ that selects a unique Green's function between the different Green's functions marking different time evolutions. For each one of the choices of the Green's function, one can reproduce the calculation from [14, 15] and see that the quasinormal modes defined by

1. the choice of the Green's function close to 0 ,

2. the outgoing radiation condition,

characterize the time evolution of the field at a fixed point within some specific time interval. Unfortunately for the case of R-N naked singularity $(f(r)=1-2 m / r+$ $\left.q^{2} / r^{2}, \quad q^{2}>m^{2}\right)$ both of the linearly independent solutions $U_{l 1}, U_{l 2}$ are regular at 0 and the problem is underdetermined. It is easy to show that both of the solutions are regular at the origin. Write (11) as:

$$
\begin{aligned}
& -f(r)^{2} \frac{d^{2} U_{l 1,2}(s, r)}{d r^{2}}-\left[\frac{f(r)(2 r-2 m)}{r^{2}}\right] \frac{d U_{l 1,2}(s, r)}{d r} \\
& +\left[s^{2}+\frac{l(l+1) f(r)}{r^{2}}\right] U_{l 1,2}(s, r)=0 .
\end{aligned}
$$

Now taking the $r \rightarrow 0$ limit of the equation (12) one obtains the following

$$
\begin{aligned}
-\frac{d^{2} U_{l 1,2}(s, r)}{d r^{2}} & +\left[\frac{2 m}{q^{2}}\right] \frac{d U_{l 1,2}(s, r)}{d r} \\
& +\frac{l(l+1)}{q^{2}} U_{l 1,2}(s, r)=0 .
\end{aligned}
$$

This means the solutions $U_{l 1,2}$ behave close to 0 as

$$
U_{l 1,2}(s, r)=\exp \left(\beta_{1,2} r\right)
$$

with

$$
\beta_{1,2}=\frac{m}{q^{2}} \pm \sqrt{\left(\frac{m}{q^{2}}\right)^{2}+\frac{l(l+1)}{q^{2}}} \in \mathbb{R},
$$

hence both are regular.

The fact that the problem is underdetermined is not surprising, since the space-time is not globally hyperbolic and anything can fall out at any time from the singularity. This means the singularity has "hair" (carries some other information beyond the metric) and the quasi-normal modes obviously depend on the "hair". Let us add here that, as we already mentioned in the introduction, the "hair" of the singularity relates to the existence of many different self-adjoint extensions of the "Hamiltonian" operator in the equation. (In 9] one can find a nice analysis of the uniqueness of the self-adjoint extensions of such operators for many different types of naked spacetime singularities including the R-N naked singularity.)

Is there any intuitive physical condition that we can further impose on the fields, that will uniquely select the appropriate Green's function? At least to get the geometrical optics continuous extension of the black hole case one can impose the condition that nothing falls in or out of the singularity. This means there is neither absorption nor superradiation in the scattering and the Smatrix of the $\mathrm{K}-\mathrm{G}$ field is a unitary operator. What does this condition mean? The conserved current 4 -vector for the complex Klein-Gordon field is given by

$$
J^{\mu}(\Psi)=-i g^{\mu \nu}\left(\Psi \nabla_{\nu} \Psi^{*}-\Psi^{*} \nabla_{\nu} \Psi\right)
$$

Let us integrate the 4-current along a cyllindrical hypersurface given by $r=r_{0}$ and $t \in\left[t_{1}, t_{2}\right]$. Then since (16) is a conserved current it holds that

$$
Q\left(t_{2}\right)-Q\left(t_{1}\right)=-\int_{\Sigma_{r_{0}}} d t d \theta d \phi \sqrt{-h} n^{\mu} J_{\mu}
$$

Here $Q\left(t_{1,2}\right)$ is the integral along a hypersurface given by the interior of the cylinder at the constant time $\left(t_{1}\right.$ or $\left.t_{2}\right)$, with a future orientated surface normal vector. $\Sigma_{r_{0}}$ is a cylindrical hypersurface given by $r=r_{0}$ and $\sqrt{-h}$ is an induced density given as $\sqrt{-h}=\sqrt{f\left(r_{0}\right)} r_{0}^{2} \sin (\theta)$. Furthermore $n^{\mu}$ is a normal vector to $\Sigma_{r_{0}}$ given as $\left.n^{(t, r, \theta, \phi)}=\left(0, \sqrt{f\left(r_{0}\right.}\right), 0,0\right)$. Since we want $Q$ to remain constant with respect to time as we take the limit $r_{0} \rightarrow 0$ (nothing flows out or into the singularity), the equation (17) reduces to the following

$$
\lim _{r_{0} \rightarrow 0} \int_{\Sigma_{r_{0}}} d t d \theta d \phi \sqrt{-h} n^{\mu} J_{\mu}=-i \lim _{r_{0} \rightarrow 0} f\left(r_{0}\right) r_{0}^{2} \int_{t_{1}}^{t_{2}} d t \int_{0}^{\pi} d \theta \sin \theta \int_{0}^{2 \pi} d \phi\left[\Psi \partial_{r} \Psi^{*}-\Psi^{*} \partial_{r} \Psi\right]=0
$$


Let us analyse the equation (18) in decomposition into spherical harmonics, hence

$$
\Psi(t, r, \theta, \phi)=\sum_{l, m} \psi_{l}(r, t) Y_{l m}(\theta, \phi) .
$$

Then we can rewrite the equation (18) as

$$
\begin{aligned}
& \lim _{r_{0} \rightarrow 0} f\left(r_{0}\right) r_{0}^{2} \sum_{l} \int_{t_{1}}^{t_{2}} d t\left[\psi_{l}(r, t) \partial_{r} \psi_{l}^{*}(r, t)\right. \\
- & \left.\psi_{l}^{*}(r, t) \partial_{r} \psi(r, t)\right]_{\mid r=r_{0}}=0,
\end{aligned}
$$

for arbitrary $t_{1}, t_{2}$. Since $f\left(r_{0}\right) r_{0}^{2} \rightarrow q^{2}$ as $r_{0} \rightarrow 0$, in order to fulfill the equation (20) we impose 2 for every $l$ and every $t$

$$
J_{r}(\Psi, 0)=\left[\psi_{l}(r, t) \partial_{r} \psi_{l}^{*}(r, t)-\psi_{l}^{*}(r, t) \partial_{r} \psi_{l}(r, t)\right]_{\mid r=0}=0 .
$$

But this means that the functions $\psi_{l}(r, t)$ should be always constrained either by the condition $\psi_{l}(0, t)=0$ or $\left.\partial_{r} \psi(r, t)\right|_{r=0}=0$. (One might argue that it will be enough to claim that the fields and their first $r$ derivatives should be real at zero, but that does not put any general constraint on the normal modes.) Now we obtain the function $\psi_{l}(r, t)$ from the normal modes as

$\psi_{l}(r, t)=\int_{0}^{\infty} d \omega e^{-i \omega t}\left[c_{1}(\omega) U_{l 1}(r, i \omega)+c_{2}(\omega) U_{l 2}(r, i \omega)\right]$,

and take the condition $\psi_{l}(0, t)=0$ for arbitrary time $t$. This translates to

$$
\int_{0}^{\infty} d \omega e^{-i \omega t}\left[c_{1}(\omega) U_{l 1}(0, i \omega)+c_{2}(\omega) U_{l 2}(0, i \omega)\right]=0 .
$$

But since both $U_{l 1}(0, i \omega)=U_{l 2}(0, i \omega)=1$ (see (14)) and Fourier-like transform given by (23) should not map non-zero functions to zero, one obtains the condition $c_{2}(\omega)=-c_{1}(\omega)$. This means we compose the relevant wave packet only from the following modes:

$$
\tilde{U}_{l}(r, i \omega)=U_{l 1}(r, i \omega)-U_{l 2}(r, i \omega) .
$$

The same line of reasoning applies to the condition $\left.\partial_{r} \psi(r, t)\right|_{r=0}=0$ and the wave-packets that fulfill such condition must be composed entirely from modes given as $(l>0)$ :

$$
\tilde{U}_{l}(i \omega, r)=\frac{U_{l 1}(i \omega, r)}{\beta_{1}}-\frac{U_{l 2}(i \omega, r)}{\beta_{2}} .
$$

For $l=0$, we have the coefficient $\beta_{2}=0$ and the solution $U_{l 2}(r, i \omega)$ behaves as a constant for $r \approx 0$ (with the value

\footnotetext{
${ }^{2}$ Such a condition on the radial part of the current at the origin (determining whether the scattering is absorptive, radiative, or superradiative) occurs in the formulation of [10], where it gives constrains on the domain of the "Hamiltonian" providing symmetricity of the operator.
}

set to 1), which means that the condition (25) does not make strictly sense; in such case the modes are given simply by the function $U_{l 2}(r, i \omega)$.

This shows that the condition of S-matrix being a unitary operator gives additional constraints on the Green's function. The previous conditions mean that we shall consider only a linear space of wave-packets formed purely from modes that vanish at zero, or a linear space of wave-packets formed purely from modes whose first $r$ derivatives vanish at zero. (Of course one cannot superpose wave-packets formed from modes having the vanishing radial derivative at 0 with wave packets formed from modes that are vanishing at 0 , since the 4 -current does not linearly depend on a wave-function.)

So let us pick one of those two types of modes, fix the particular Green's function and employ the following reasoning: Since $J_{0}$ is conserved and the coefficients in the equation (5) are everywhere outside 0 a smooth function of $r$, the function $\psi(r, t)$, arising from compactly supported initial data could be unbounded in time only in the case the wave-packet becomes concentrated around the singularity and slowly growing asymptotically with time into delta function

$$
\lim _{t \rightarrow \infty} \psi(r, t) \sim \delta(r)
$$

But this scenario is prevented by the boundary condition that "nothing flows in or out of the singularity at any time". This means we expect the solution to be bounded with respect to both time and space and all the reasoning based on the assumption of the existence of Laplace transform is justified.

Further in the text we will employ the field vanishing condition at 0 . (This boundary condition at the singularity corresponds to what is known as Friedrich's extension of a symmetric operator.) Thus the quasinormal modes will relate to the scattering problem following from the time evolution determined by the boundary condition $\psi(0, t)=0$. But everything that we will do in the following text can be repeated for any other meaningful normal modes boundary condition giving another time evolution of the fields. The general dependence of the quasi-normal modes (characteristic oscillations) on such a boundary condition (given by some linear combination $\left.U_{l 1}(r, i \omega)+K U_{l 2}(r, i \omega)\right)$ describes the way the quasi-normal modes depend on the "hair" of the singularity. Since the quasi-normal modes are the ones that carry astrophysical information about the astrophysical sources, their dependence on the "hair" of the singularity (in some simplifying sense given by the complex parameter $K$ ) has a potential astrophysical importance (of course in case naked singularities have any astrophysical importance). 


\section{THE SCALAR WAVE SCATTERING ON A NAKED SINGULARITY}

Using $\phi_{l}$ defined as $\phi_{l}(r, t)=r \psi_{l}(r, t)$ and $x$ the tortoise coordinate given by the condition:

$$
\frac{d r}{d x}=f(r)
$$

one can rewrite the equation (5) into the following form

$$
\frac{\partial^{2} \phi_{l}(x, t)}{\partial t^{2}}-\frac{\partial^{2} \phi_{l}(x, t)}{\partial x^{2}}=V(m, q, l, x) \phi_{l}(x, t),
$$

with

$$
V(m, q, l, x)=\left[\frac{l(l+1)}{r^{2}(x)}+\frac{2 m}{r^{3}(x)}-\frac{2 q^{2}}{r^{4}(x)}\right] f(r(x)) .
$$

And, for the normal modes $e^{-i \omega t} \phi_{l}(r)$, we can write

$$
\frac{\partial^{2} \phi_{l}(x)}{\partial x^{2}}+\left[\omega^{2}-V(m, q, l, x)\right] \phi_{l}(x)=0 .
$$

If $|q|>m$, we can see that $f(r)$ given by eq. (3) has no zeros for real arguments, but eq. (27) can still be integrated to give

$$
\begin{aligned}
x=r & +\frac{2 m^{2}-q^{2}}{\sqrt{q^{2}-m^{2}}} \arctan \left(\frac{r-m}{\sqrt{q^{2}-m^{2}}}\right) \\
& +m \ln \left(r^{2}-2 m r+q^{2}\right)+C,
\end{aligned}
$$

where $C$ is an integration constant. We remark here $r_{*} \rightarrow$ $\infty$ as $r \rightarrow \infty$, but for $r \rightarrow 0$ we have

$$
\begin{aligned}
x(r \rightarrow 0) & =\frac{2 m^{2}-q^{2}}{\sqrt{q^{2}-m^{2}}} \arctan \left(\frac{-m}{\sqrt{q^{2}-m^{2}}}\right) \\
& +m \ln \left(q^{2}\right)+C=x(0)=\text { constant }
\end{aligned}
$$

as can be seen in fig. (11). Further in the text we take the tortoise coordinate with the boundary condition $x(0)=0$. It means that with respect to the usual $(C=0)$ tortoise coordinate such tortoise coordinate is shifted to the origin by the transformation $x \rightarrow x(r)-x(0)$.

The potential (29) has for the ratio $q^{2} / m^{2}$ less than approximately $9 / 8$ and the relevant $x$ (in the naked singularity case the domain of $x$ is constrained) 3 extrema, one smaller "outer" maximum, one dominant "inner" maximum and minimum in the potential valley between them. (For $r \rightarrow 0$ the function $V(r) \rightarrow-\infty$.) For $q^{2} / m^{2}$ more than approximately $9 / 8$ the potential has only one maximum (thus only one peak). These features of the potential (29) can be seen in the figure 2.

Moreover, in the case $q^{2} / m^{2}$ less than approximately $9 / 8$ and $l \gg 1$, the inner maximum becomes completely dominant, making the outer peak negligible as compared to the size of the inner peak. For the outer peak and for $l \gg 1$ the terms $\frac{2 m}{r^{3}}-\frac{2 q^{2}}{r^{4}}$ represent only a small correction (as compared to the term proportional to $l^{2}$ )

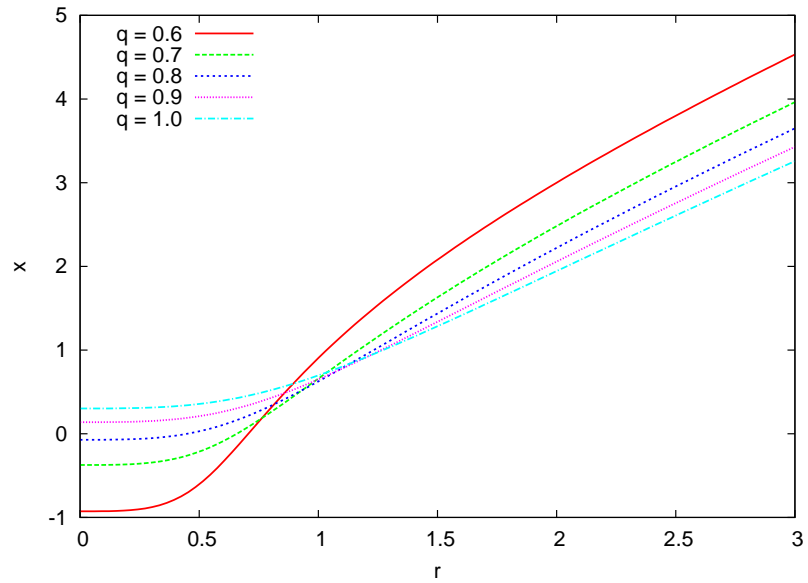

FIG. 1. Typical examples of the behavior of the new tortoise coordinate defined by eq. (31) for a spacetime with $m=0.5$ and different values of $q$, taking $C=0$.

and the second peak will vanish in this approximation at 3 $q^{2} / m^{2}=9 / 8$. (This is because the outer peak lies always at $r>1$. It is also quite obvious that the inner peak exists only due to the fact that the terms $\frac{2 m}{r^{3}}-\frac{2 q^{2}}{r^{4}}$ become dominant for $r$ close to 0 .) The features described in this paragraph can be observed in the figure 3 .

Further in the text we will use the following notation related to the potential parameters: By $V_{1}, V_{2}$ we mean the heights of the two peaks. ( $V_{1}$ is the first larger peak, $V_{2}$ the second smaller peak.) By $\alpha_{1}$ we call the curvature of the first peak and by $\alpha_{2}$ the curvature of the second peak. Furthermore, by $x_{1 \max }$ we mean the point of the location of the top of the first peak and by $x_{2 \max }$ the point of the location of the second peak. Many of these parameters can be exactly calculated in the $l \gg 1$ limit. The results of these calculations are given in the appendix A.

\section{EIKONAL LIMIT - THE PARTICLE PICTURE}

First let us have a look at the geometrical optics (eikonal) limit of the Klein-Gordon equation, which is valid for $l \gg 1$. In particular what does the geometrical optics limit tell us about the fundamental mode? The effective potential for the massless particle is

$$
V(r)=\frac{l^{2}}{r^{2}} f(r)
$$

\footnotetext{
3 As trivially expected, we will see that the same result comes directly from the potential for the motion of a massless particle.
} 


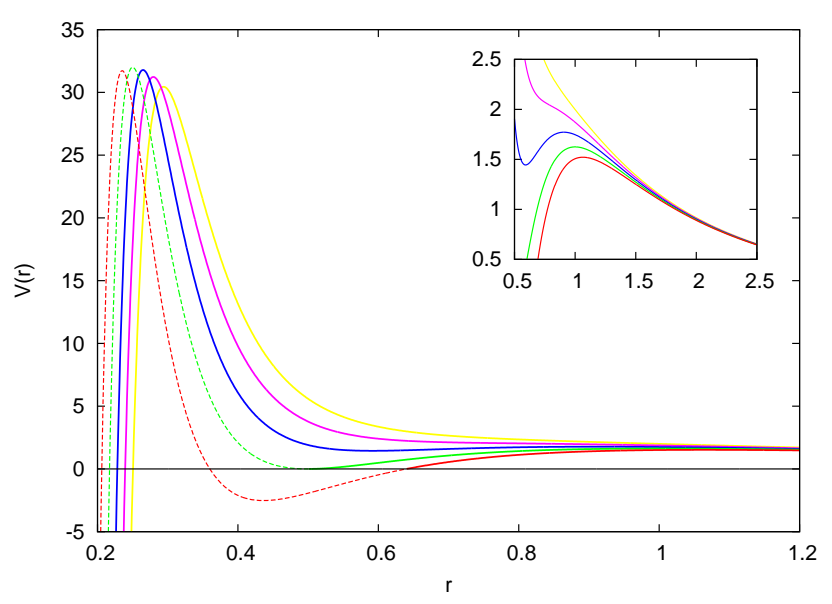

FIG. 2. Potential $V(r)$ given by eq. (29) with $l=2, m=0.5$ for $q=0.48,0.5,0.52,0.54$ and 0.56 . (The curves from left to right correspond to the increase of charge.) Note that the dashed part of the potential (for $q=0.48$ and 0.5 ) is inside the black hole horizon.

The effective potential goes to plus infinity as $r \rightarrow 0$ and its extremes are given as

$$
r_{1,2}=\frac{3 m \pm m \sqrt{9-8 \frac{q^{2}}{m^{2}}}}{2} .
$$

This means the potential has one minimum and one maximum for

$$
\frac{q^{2}}{m^{2}}<\frac{9}{8}
$$

For

$$
\frac{q^{2}}{m^{2}} \geq \frac{9}{8}
$$

there are no extrema. The maximum represents the unstable orbit of a massless particle, and for the black hole case it is only the maximum that is relevant (because it is located above the horizon). For the naked singularity with $q^{2} / m^{2} \leq 9 / 8$ there exists also a stable orbit of a massless particle. However the fundamental mode in the geometrical optics limit is related to the unstable orbit as

$$
\omega=\sqrt{V\left(r_{\max }\right)}-\frac{i}{2} \sqrt{-\frac{V^{\prime \prime}\left(r_{\max }\right) f^{2}\left(r_{\max }\right)}{2 V\left(r_{\max }\right)}}
$$

(For a very good paper that discusses this topic see [17].) Note that here $\sqrt{-\frac{V^{\prime \prime}\left(r_{\max }\right) f^{2}\left(r_{\max }\right)}{2 V\left(r_{\max }\right)}}$ gives the unstable orbit decay rate and furthermore holds the following

$$
\begin{aligned}
V^{\prime \prime}\left(r_{\max }\right) f^{2}\left(r_{\max }\right) & \left.\equiv\left(\frac{\partial^{2} V(r)}{\partial r^{2}} f^{2}(r)\right)\right|_{r_{\max }} \\
& =\left.\frac{\partial^{2} V(x)}{\partial x^{2}}\right|_{x_{\max }} .
\end{aligned}
$$

(Again $x$ is the tortoise coordinate.) Particularly for the Reissner-Nordström black hole/naked singularity (with $q^{2} / m^{2}<9 / 8$ ) equation (37) can be expressed as:

$$
\omega=\frac{l}{r_{\max }} \sqrt{1-\frac{2 m}{r_{\max }}+\frac{q^{2}}{r_{\max }^{2}}}-\frac{i}{2 r_{\max }} \sqrt{-3+\frac{18 m}{r_{\max }}-\frac{13 q^{2}+24 m^{2}}{r_{\max }^{2}}+\frac{32 m q^{2}}{r_{\max }^{3}}-\frac{10 q^{4}}{r_{\max }^{4}}}
$$

with $r_{\max }=\frac{3 m+\sqrt{9 m^{2}-8 q^{2}}}{2}$, given as before.

From the black hole QNM-s boundary conditions one can see that such massless particles decaying from their unstable orbit and eventually escaping either to the B-H horizon, or the infinity represent the fundamental QNM$\mathrm{s}$ in the $l \gg 1$ (geometrical) limit. One can observe (see the discussion later) that the same holds in the naked singularity case. In the $l \gg 1$ case the fundamental mode of the massless perturbations is described by the picture of a particle decaying from its unstable "photon" orbit. (Note that in the naked singularity case the stable photon orbit makes the particle decaying inwards oscillate around the stable orbit.) This should suggest that in the geometrical optics limit

1. one shall expect continuity of the fundamental mode (as a function of $q, m$ ) when turning from the black hole to the naked singularity case,

2. for $q^{2} / m^{2} \geq 9 / 8$ there is a clear indication that there do not exist low damped quasi-normal modes.

The way how to understand the continuity (for $l \gg 1$ ) of the fundamental mode as one transfers from the black hole case to the naked singularity case could be the following: Consider the partial S-matrix $S_{l}(\omega)$. Within the S-matrix one can look for poles on the complex plane: obviously any of the poles must be necessarily non-real. For the relativistic time dependence convention chosen as $\exp (-i \omega t)$ the poles with $\omega_{I}>0$ must be purely imaginary (as a result of the given Hamiltonian being a symmetric operator) and they represent bound states with the non-relativistic energies given as $E=-\omega_{I}^{2}$. The poles 

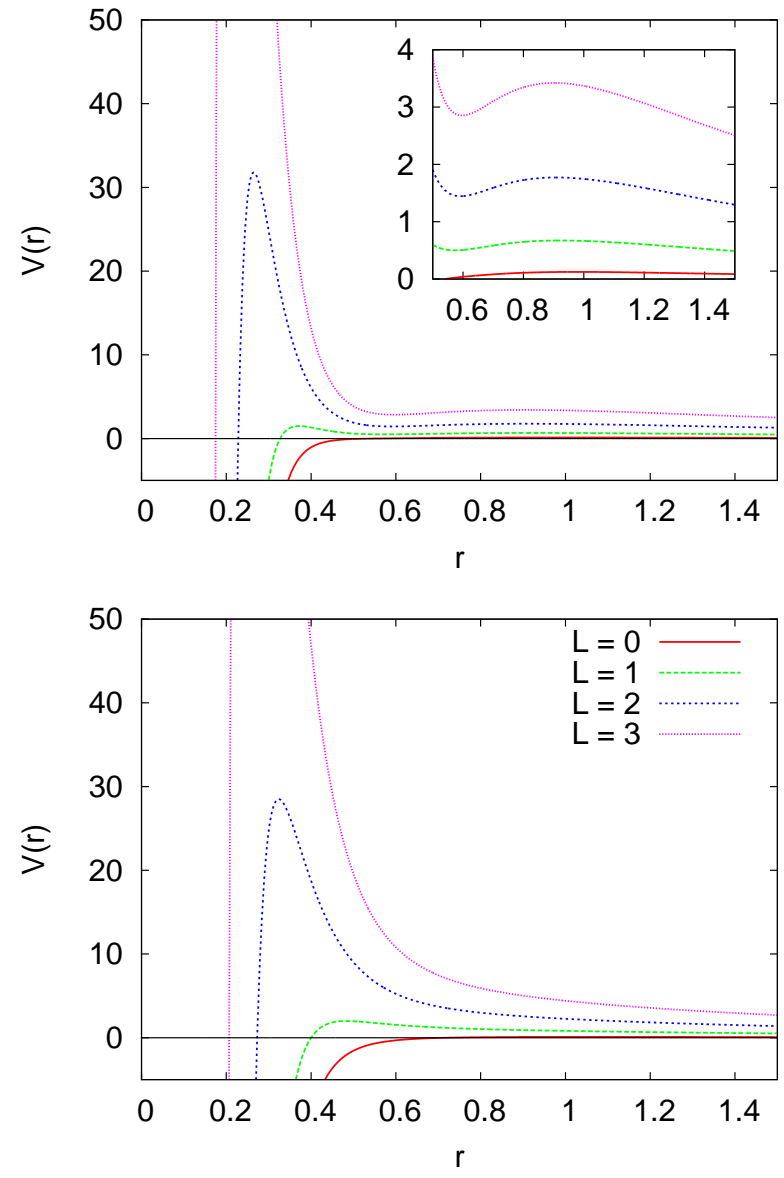

FIG. 3. Above: potential $V(r)$ given by eq. (29) with $m=0.5$ for $q=0.52\left(q^{2} / m^{2}<9 / 8\right)$ and different values of $l$ (same caption as on the right plot). Note in this case the existence of the secondary peak, reminiscent of the black hole potential. Below: the same, but this time for $q=0.6\left(q^{2} / m^{2}>9 / 8\right)$. There is no secondary peak in this case.

with $\omega_{I}<0$ represent quasinormal frequencies. Some of the quasinormal frequencies correspond to the resonances in the phase factor related to the partial S-matrix, and the wave packets formed out of the resonance energies represent quantum particles tunnelling out of the potential valley; for the non-relativistic quantum particles (for example) with the decay rate given as $\left(\omega^{2}\right)_{I}=2 \omega_{R} \omega_{I}$. Now in the naked singularity case the fundamental mode is represented by a resonance that has a low enough energy (at the level of the smaller peak) and gets trapped in the potential valley in between the peaks for some time and then radiated away. The larger peak behind the smaller peak is hugely dominant (large $l$ ) and effectively acts to the wave packet as an infinite barrier. There are probably much higher resonance energies related to higher QNM frequency overtones that are determined by the higher peak, but left unaffected by the details of the small peak. So there are two different effective regimes for the quasinormal modes, in the first regime they are sensitive only to the details of the smaller peak, in the second regime they are sensitive only to the details of the larger peak. This splitting disappears in the case of small $l$-s since the two peaks are of comparable heights and effectively interfere.

Let us finish this section with one more remark: In the black hole case one can relate the fundamental mode to the peak of the potential (independently of $l \gg 1$ ) following the way of thinking in [18]. The ratio of the amplitudes of the reflected and transmitted waves $A_{\text {ref }}(\omega) / A_{\text {trans }}(\omega)$ is for quasinormal modes 1 . The same happens for real $\omega$-s, when the energies $\omega^{2}$ are close ("almost at") to the peak of the potential. So for "almost" real $\omega^{2}$ (fundamental mode) the continuity of the ratio of reflected and transmitted amplitudes analytically continued to the complex plane suggests that the real part of the frequency should be near the square root of the peak of the potential. (This intuition is then to some extend confirmed also semi-analytically, such as by the Ferrari and Mashhoon approach using the Poeschl-Teller potential [19], or by using the inverted harmonic oscillator potential.) In the case of a naked singularity life is not so easy (in the reduced 1D problem we have no transmitted amplitudes), so this simple logic fails. (One can say luckily it fails as if it remained valid one would also for $l \gg 1$ expect a discontinuity in the fundamental mode as one jumps from the black hole to the naked singularity. This is because the second, dominant peak suddenly appears in the domain of the $x$ coordinate, due to the fact that the domain of $x$ discontinuously jumps when passing from the black hole to the naked singularity case.) Certainly one can say that any of the quick fits, such as were done in the black hole case do not lead to anything close to the numerical data obtained in this paper.

\section{GENERAL ANALYTICAL METHODS TO MODEL THE PROBLEM}

In this section we want to suggest some ways to model the naked singularity scattering (with a general time evolution) by solutions of the equation (30) with $V(x, l, m, q)$ replaced by analytically solvable potentials. The reasons are the following: First, this section serves as a basis for the analysis of the $l \gg 1$ cases provided in the section VI. Second, it demonstrates that the naked singularity scattering is in principle treatable via analytical approximations and thus shows the general power of analytical techniques. (Also the approximations obtained here might produce some future results for the quasinormal frequencies. For some further calculations see the appendix B, Furthermore, for a very nice overview of the results for the quasi-normal frequencies and related transmission resonances of the analytically tractable potentials see [21].) Third, it might bring more insights into the physics obtained through the exact scalar field 
potential (29).

The key point is to split the scalar field potential into different domains and approximate it on each of those domains (or directly the solution of the equation (30) ) by a different, analytically solvable potential. Then one has to impose the standard procedure: The logarithmic derivatives of the solutions on different domains must be glued on the domain's boundary. We might add here that since the analytically solvable potentials are typically fitted by the parameters of the peaks one expects such approximation to work for the modes that are not too damped (the low damped QNM modes). The approximation cannot be taken too seriously for the highly damped modes.

Let us suggest modeling all the solutions of the relevant cases as follows:

\section{The case $q^{2} / m^{2} \gtrsim 9 / 8$ for arbitrary $l$}

For this case it might be interesting to consider the Morse potential. (For the definition and origins of all the potentials used in this section see also [21].) On the other hand the infinite valley behind the one and only peak may not be well modelled by only a finite valley that is behind the peak of the Morse potential. Thus we consider the solution in the region "behind" Morse potential region to be given as (24) for $x \rightarrow 0$ and the logarithmic derivative gluing condition originates from:

1. $0<x<a_{1}$ : use function given as (for the details see equation (24)):

$$
\phi(r)=r \Psi(r)=A r\left(e^{\beta_{1} r}-e^{\beta_{2} r}\right) .
$$

2. $a_{1} \leq x$ : use the appropriate solution of the equation (30) with the Morse potential:

$$
V_{1} e^{-\alpha_{1}\left(x-x_{1 \max }\right)}\left(2-e^{-\alpha_{1}\left(x-x_{1 \max }\right)}\right) .
$$

Here $a_{1}$ is a point most conveniently chosen where the Morse potential is 0 (for any arbitrary $l$ there is such point for $x>0$ ). This means $a_{1}$ can be analytically given as

$$
a_{1}=x_{1 \max }-\frac{1}{\alpha_{1}} \ln (2) .
$$

Let us make here one remark: Instead of approximating the original scalar field equation for $r \approx 0$ one can consider taking the equation (30) in the $r \rightarrow 0$ approximation. This leads to the following:

$$
\frac{d^{2} \phi(x)}{d x^{2}}+\frac{2}{9} \frac{\phi(x)}{x^{2}}=0, \quad x \rightarrow 0 .
$$

The equation (42) has solutions $\phi=C_{1} x^{1 / 3}+C_{2} x^{2 / 3}$. The solution such that fulfills $\Psi(0)=0$ must have $C_{1}=$ 0 . This approximation of the solution we think to be less exact as the approximation given as (40), as it is in fact effectively only the first non-zero term of the power series expansion of (40) taken at 0 . (For such reasons we decided to use in our analytical approximations the approximate solution (40).)

\section{The case of $q^{2} / m^{2} \gtrsim 9 / 8$ in the large $l$ limit}

Let us consider now the limit of large $l$. In this case, since the peak already lies close to 0 and the change in the highest power of inverted $x$ becomes more and more dominant (with higher $l$-s), one might simplify the approximation given in case V1, by using the following potential:

1. $0<x<x_{1 \max }$ : use function given as (40)

2. $x_{1 \text { max }} \leq x$ : use the appropriate solution of the equation (30) with the potential (Poeschl-Teller potential):

$$
V_{1} \cosh ^{-2}\left[\alpha_{1}\left(x-x_{1 \max }\right)\right] .
$$

Here we also used the Poeschl-Teller potential instead of the Morse potential, because the logarithmic derivative gluing condition is much easier to solve for the solutions of the Poeschl-Teller than for the solutions of the Morse potential. The expense of the simplicity is that the approximation by such potential might be slightly less exact than by using the Morse potential, but one still expects it to be accurate enough.

\section{The case $q^{2} / m^{2} \lesssim 9 / 8$ for arbitrary $l$}

If one does not want to neglect the second smaller peak (so one is interested in resonances related to the valley between the two peaks), the analytically treatable potential describing all the resonances (and also higher damped QNM frequencies) could be:

1. $0<x<a_{1}$ : use function given as (40),

2. $a_{1} \leq x \leq a_{2}$ : solutions of the equation (30) with the Morse potential

$$
V_{1} e^{-\alpha_{1}\left(x-x_{1 \max }\right)}\left(2-e^{-\alpha_{1}\left(x-x_{1 \max }\right)}\right)
$$

3. $a_{2} \leq x$ : solution of the equation (30) with the Poeschl-Teller potential:

$$
V_{2} \cosh ^{-2}\left[\alpha_{2}\left(x-x_{2 \max }\right)\right] .
$$

Here $a_{1}$ is best chosen as in the case V1 and $a_{2}$ is chosen to be such that the resulting potential is continuous. Generally $a_{2}$ has to be obtained numerically, but for larger $l$-s (certainly $l=20$ is more than enough, as we checked), the curvature of the second peak is very small comparing to the curvature of the first peak and also the 
curvature of the first peak becomes (for large $l$-s) very large comparing to the scales of the potential, so that one can calculate (for $l=20$ with a good approximation at least to the 6 decimal places) $a_{2}$ just by

1. taking the Poeschl-Teller potential to be constant and given by the hight of the outer peak

2. simplifying the Morse potential by the following approximation:

$$
V(x) \approx 2 V_{1} e^{-\alpha_{1}\left(x-x_{1 \max }\right)} .
$$

The resulting formula then becomes

$$
a_{2}=x_{1 \max }-\frac{1}{\alpha_{1}} \ln \left(\frac{V_{2}}{2 V_{1}}\right) .
$$

Then one can easily show that $a_{2} \rightarrow 0$ as $l \rightarrow \infty$.

\section{The case of $q^{2} / m^{2} \lesssim 9 / 8$ in the large $l$ limit}

Now consider what happens with the resonances related to the valley between the two peaks in the large $l$ limit. In such case the dominant peak grows to infinity as compared to the smaller peak, the infinite valley behind the dominant peak shrinks to 0 . Also $a_{1} \rightarrow 0$ and the valley between the two peaks becomes flat as compared to the difference between the height of the first peak and the value of the potential at the bottom of the valley. If the resonances related to the smaller peak locate close to the top of the peak (they should since the first peak grows with $l$ an makes the tunnelling harder), then one might effectively approximate the case $\mathrm{V3}$ by the Poeschl-Teller potential:

$$
V_{2} \cosh ^{-2}\left[\alpha_{2}\left(x-x_{2 \max }\right)\right],
$$

for $0 \leq x$. (One can also derive this approximation straight from the logarithmic derivative gluing condition in the appendix B2 by taking the $l \rightarrow \infty$ limit.)

\section{THE LARGE $l$ LIMIT - THE ANALYTIC APPROACH}

In this section we want to analytically confirm the results obtained directly from the eikonal limit.

\section{Calculations for $q^{2} / m^{2}<9 / 8$}

The case V4 from the previous section is easily solvable. The solution in the region $x \geq 0$ must fulfil the outgoing radiation condition. (To be exact after analytically extending the solution to the complex plane it must give asymptotically the outgoing waves on the line $(\omega x)_{I}=0$.) Such solution is given as follows

$$
\phi_{R}(x)=C_{2} e^{i \omega x} F_{21}\left(g_{1}, g_{2}, g_{3},\left(1+\exp \left(2 \alpha_{2}\left(x-x_{2 \max }\right)\right)\right)^{-1}\right),
$$

where $F_{21}$ is the standard hypergeometric function (of the type 2-1) and

$$
\begin{aligned}
& g_{1} \equiv \frac{1}{2}+\sqrt{\frac{1}{4}-\frac{V_{2}}{\alpha_{2}^{2}}} \equiv-g_{2}+1 \\
& g_{3} \equiv 1-\frac{i \omega}{\alpha_{2}} .
\end{aligned}
$$

The boundary condition at 0 gives $\phi_{R}(0)=0$, leading to

$$
F_{21}\left(\frac{1}{2}+\frac{i \sqrt{V_{2}}}{\alpha_{2}}, \frac{1}{2}-\frac{i \sqrt{V_{2}}}{\alpha_{2}}, 1-\frac{i \omega}{\alpha_{2}},\left(1+\exp \left(-2 \alpha_{2} x_{2 \max }\right)\right)^{-1}\right)=0
$$

Unless $q^{2} / \mathrm{m}^{2}$ is not too close to the upper limit given for $l$ large as $9 / 8$, one can reliably approximate $e^{-2 \alpha_{2} x_{2 \max }} \approx 0$ and the condition (52) turns to be:

$$
F_{21}\left\{\frac{1}{2}+\frac{i \sqrt{V_{2}}}{\alpha_{2}}, \frac{1}{2}-\frac{i \sqrt{V_{2}}}{\alpha_{2}}, 1-\frac{i \omega}{\alpha_{2}}, 1\right\}=0
$$

Now (53) can be rewritten through Gamma functions and reduces to the simple problem of finding poles of the product of Gamma functions

$$
\Gamma\left[-\frac{i \omega-i \sqrt{V_{2}}}{\alpha_{2}}+\frac{1}{2}\right] \Gamma\left[-\frac{i \omega+i \sqrt{V_{2}}}{\alpha_{2}}+\frac{1}{2}\right]=0 .
$$

The poles are located at $-n$ for $n$ being a natural number and this gives

$$
\omega= \pm \sqrt{V_{2}}-i \alpha_{2}\left(n+\frac{1}{2}\right) .
$$


This is precisely the formula for the lowest damped black hole QNM frequencies. This means in the $l \gg 1$ limit we see (at least for the lowest modes) a continuous transition from the black hole case to the naked singularity case, as expected. (At least in the case where it holds that $e^{-2 \alpha_{2} x_{2 \max }} \approx 0$, which becomes a less accurate approximation for $q^{2} / m^{2}$ close to $9 / 8$. On the other hand for such ratios of $q^{2} / \mathrm{m}^{2}$ the peak is almost vanished and one can assume that the decaying circular orbit eikonal picture has already quite limited sense.)

All this means that in the regime $l \gg 1$ the lowest QNM frequencies should be located close to the first peak of the potential and are (up to certain $n$ ) given by the formula (55). They represent resonances such that they are insensitive to the details of the larger peak as in their case the larger peak can be already seen to act effectively as an infinite potential barrier. Hence we can conclude that in the $l \gg 1$ case we are able to match the geometrical (eikonal) limit of the original problem considered.

\section{Calculations for $q^{2} / m^{2} \geq 9 / 8$}

Take the case $\mathrm{V} 2$ from the previous section. On the left hand side of the logarithmic derivative gluing condition we need a logarithmic derivative of the function:

$$
r \Psi_{L}=C_{L} r\left(e^{\beta_{1} r}-e^{\beta_{2} r}\right) .
$$

On the right hand side of the logarithmic derivative gluing condition we need the logarithmic derivative of the solution on the interval $\left[x_{1 \max }, \infty\right]$. It is given as in (49), only the parameters are related to the first and only peak in this case. Then the logarithmic derivative gluing condition leads to the following:

$$
\frac{\Gamma\left(-\frac{i \omega}{2 \alpha_{1}}-\frac{1}{2} \sqrt{\frac{1}{4}-\frac{V_{1}}{\alpha_{1}^{2}}}+\frac{3}{4}\right) \Gamma\left(-\frac{i \omega}{2 \alpha_{1}}+\frac{1}{2} \sqrt{\frac{1}{4}-\frac{V_{1}}{\alpha_{1}^{2}}}+\frac{3}{4}\right)}{\Gamma\left(-\frac{i \omega}{2 \alpha_{1}}-\frac{1}{2} \sqrt{\frac{1}{4}-\frac{V_{1}}{\alpha_{1}^{2}}}+\frac{1}{4}\right) \Gamma\left(-\frac{i \omega}{2 \alpha_{1}}+\frac{1}{2} \sqrt{\frac{1}{4}-\frac{V_{1}}{\alpha_{1}^{2}}}+\frac{1}{4}\right)}=\frac{f\left(r_{\max }\right)}{2 \alpha_{1}}\left[\frac{1}{r_{\max }}+\frac{\beta_{1} e^{\beta_{1} r_{\max }}-\beta_{2} e^{\beta_{2} r_{\max }}}{e^{\beta_{1} r_{\max }}-e^{\beta_{2} r_{\max }}}\right]
$$

If one takes the $l \rightarrow \infty$ limit then the equation (57) becomes:

$$
\frac{\Gamma\left(-i \tilde{\omega}+\frac{1}{2 \sqrt{6}}+\frac{3}{4}\right) \Gamma\left(-i \tilde{\omega}-\frac{1}{2 \sqrt{6}}+\frac{3}{4}\right)}{\Gamma\left(-i \tilde{\omega}+\frac{1}{2 \sqrt{6}}+\frac{1}{4}\right) \Gamma\left(-i \tilde{\omega}-\frac{1}{2 \sqrt{6}}+\frac{1}{4}\right)}=\frac{1}{4}\left[\frac{1}{\sqrt{3}}+\operatorname{coth}(\sqrt{3})\right]
$$

Here $\tilde{\omega}$ is defined as $\tilde{\omega}=\omega /\left(2 \alpha_{1}\right)$. For not highly damped frequencies in the $l \gg 1$ limit it must hold that $\tilde{\omega} \in \mathbb{R}$. But by plotting the right side of the equation (58) minus the left side of the equation (58) in Mathematica it can be shown that the equation (58) does not have any real solutions. For more than large intervals of values of $\tilde{\omega}$ the absolute value of the left hand side of the equation minus the right hand side of the equation always seems to grow (almost) linearly with respect to $\tilde{\omega}$ from the value approximately given as 0.35 at $\tilde{\omega}=0$ to infinity. It might not be hard to prove also analytically that (58) does not have real solutions by using many relatively simple properties of Gamma functions. This means there are no low damped modes in the case of $q^{2} / m^{2}>9 / 8$ and $l \gg 1$, as expected from the eikonal limit / particle picture. (It means the damping of the fundamental mode grows to infinity as $l \rightarrow \infty$ and grows no less rapidly than $\alpha_{1}$, hence no less rapidly than cubically with $l$.)

\section{THE NAKED SINGULARITY FOR THE SMALL WAVE MODE NUMBERS - NUMERICAL RESULTS FOR THE FREQUENCIES}

In [22], the quasi-normal modes of a scalar field in an electrically charged Vaidya background were studied using a similar numerical setup to what we are going to use here. An interesting investigation done in [22] tried to determine what would happen with the quasi-normal modes as the time-dependent background approached a naked singularity, but the numerical code used was not suitable for following the field evolution after the extremal configuration $(q=m)$ was reached. In our present work, we have a static configuration that describes a naked a singularity in order to study the properties of the quasinormal modes.

Our objective in this section is to solve eq. (28) with potential (29) numerically, in the case where $q>m$ as described in the last section. To do this, we rewrite eq. (28) in terms of the light-cone variables $u=t-x$ and $v=t+x$, where $x$ corresponds to the tortoise coordinate (27), as

$$
\frac{\partial^{2} \phi}{\partial t^{2}}-\frac{\partial^{2} \phi}{\partial x^{2}}=-4 \frac{\partial^{2} \phi}{\partial u \partial v}=V(r) \phi
$$


that can be integrated with the boundary conditions

$$
\begin{aligned}
& \phi(r=0, t)=\phi\left(u, v=u+2 x_{0}\right)=0, \\
& \phi(u=0, v)=e^{-\frac{\left(v-v_{c}\right)^{2}}{2 \sigma^{2}}},
\end{aligned}
$$

where condition (60) is a necessary condition on the field $\phi$ near the origin (see the discussion on fig. 5] below) and condition (61) defines an "arbitrary" relevant initial signal to be propagated. We use the algorithm

$$
\phi_{N}=\phi_{W}+\phi_{E}-\phi_{S}-\frac{\phi_{W}+\phi_{E}}{8} V \Delta_{v} \Delta_{u}
$$

see where $\Delta_{u}$ and $\Delta_{v}$ are the integration steps in $u$ and $v$, respectively, and the definitions of $\phi_{N}$ etc can be seen on fig. 4. Note that here $V$ is the potential (29) evaluated at the same $r$ coordinate as $\phi_{S}\left(\right.$ and $\left.\phi_{N}\right)$.

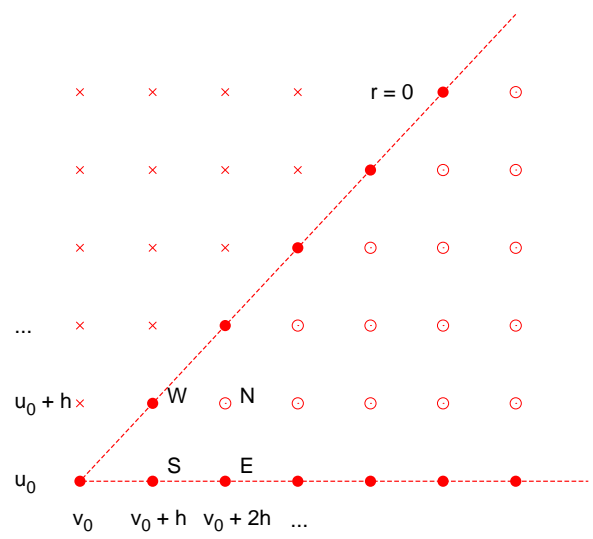

FIG. 4. Example of the numerical grid used for integrating eq. (59) with boundary conditions (60) and (61). The points marked with " $x$ " are out of our domain, values of $\phi$ at the positions marked with filled circles are given by the boundary conditions and the values at the empty circles are obtained with the algorithm (62).

As we can see in fig. 5, the boundary conditions (60) and (61) ensure the necessary conditions on the fields $\phi$ and $\psi$ near the center. As we discussed previously in section [II, the physically correct boundary condition for $\psi$ is $\psi(0, t)=0$. From this we must have for $\phi(r, t)=$ $r \psi(r, t)$ that $\phi(0, t)=0$ and $\phi^{\prime}(0, t)=0$. In fact, the quantity

$$
\mathcal{E}=\int\left[\left(\frac{\partial \phi}{\partial t}\right)^{2}+\left(\frac{\partial \phi}{\partial x}\right)^{2}+V(r) \phi^{2}\right] d x
$$

is invariant along the $t$-evolution governed by (59) with the boundary conditions $\phi(r=0, t)=\phi(r \rightarrow \infty, t)=0$. Taking into account (27), the integrand can be expressed near $r=0$ as

$$
\frac{q^{4}}{r^{4}}\left(\left(\phi^{\prime}\right)^{2}-\frac{2}{r^{2}} \phi^{2}\right)
$$

with the prime denoting the derivative with respect to $r$. Any initial condition with finite $\mathcal{E}$, as those ones obeying (60) and (61), is such that

$$
\left(\phi^{\prime}\right)^{2}-\frac{2}{r^{2}} \phi^{2} \sim r^{2+\alpha},
$$

for $r \rightarrow 0$, with $\alpha>0$, implying that $\phi^{\prime}(r=0, t)=0$ for initial condition with finite E obeying $\phi(r=0, t)=0$. Moreover, we have checked that our boundary conditions for $\phi$ also reproduce the $\phi^{\prime}(0, t)=0$ condition, by testing the code with the condition $\phi(\bar{r}, t)=0$, where $\bar{r}$ is a very small value, and we found no differences in the late time evolution of the fields.
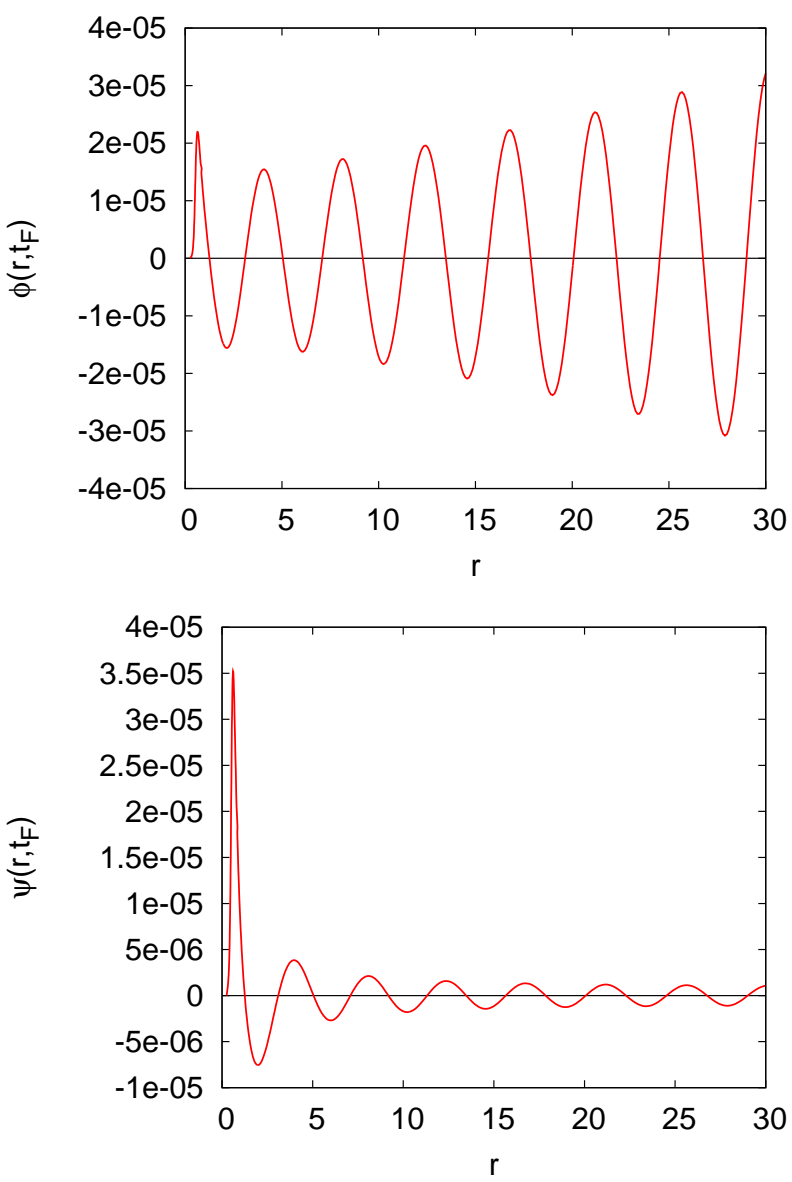

FIG. 5. Above: Behavior of $\phi$ with $l=2$ as a function of $r$ near the center $r=0$ for a late time $t_{F}=350$, shown here in order to exemplify the effect of conditions (60) and (61) in the numerical integration, for a spacetime with $q=0.5$ and $q=0.52$. Below: The same as in the left plot, but this time for the function $\psi=\phi / r$.

In the left plot of fig. 6 we present some typical time evolutions of $\phi$, for a $l=2$ and different $q / m$ ratios. In the right plot we present the obtained frequencies of the QNMs (fundamental mode) in the $\omega_{R} \times \omega_{I}$ plane. We can see a discontinuity in the frequencies as $q / m \rightarrow 1$, as 
was expected from the discussion of the potential $V(r)$ (see fig. 2). We also point here that we see no significant changes, but rather a smooth behavior as $q^{2} / m^{2} \rightarrow 9 / 8$ $(q / m \rightarrow 1.06$ in the plot). But we see a point of inflection in $\omega_{R}$ at $q / m \approx 1.16$, for which we did not find an analytical explanation.

We remark here some numerical issues that prevented us from extending the results shown in fig. 6] for lower and higher $q / m$ rations. As $q / m \rightarrow 1$ and $\phi$ becomes less damped, different modes subsist for longer times and and longer evolutions are needed in order to obtain the clear frequency of the fundamental mode. On the other hand, as we increase $q / m, \phi$ is damped so quickly that we cannot observe enough oscillation cycles to obtain the frequencies. These are the issues that have limited our results.
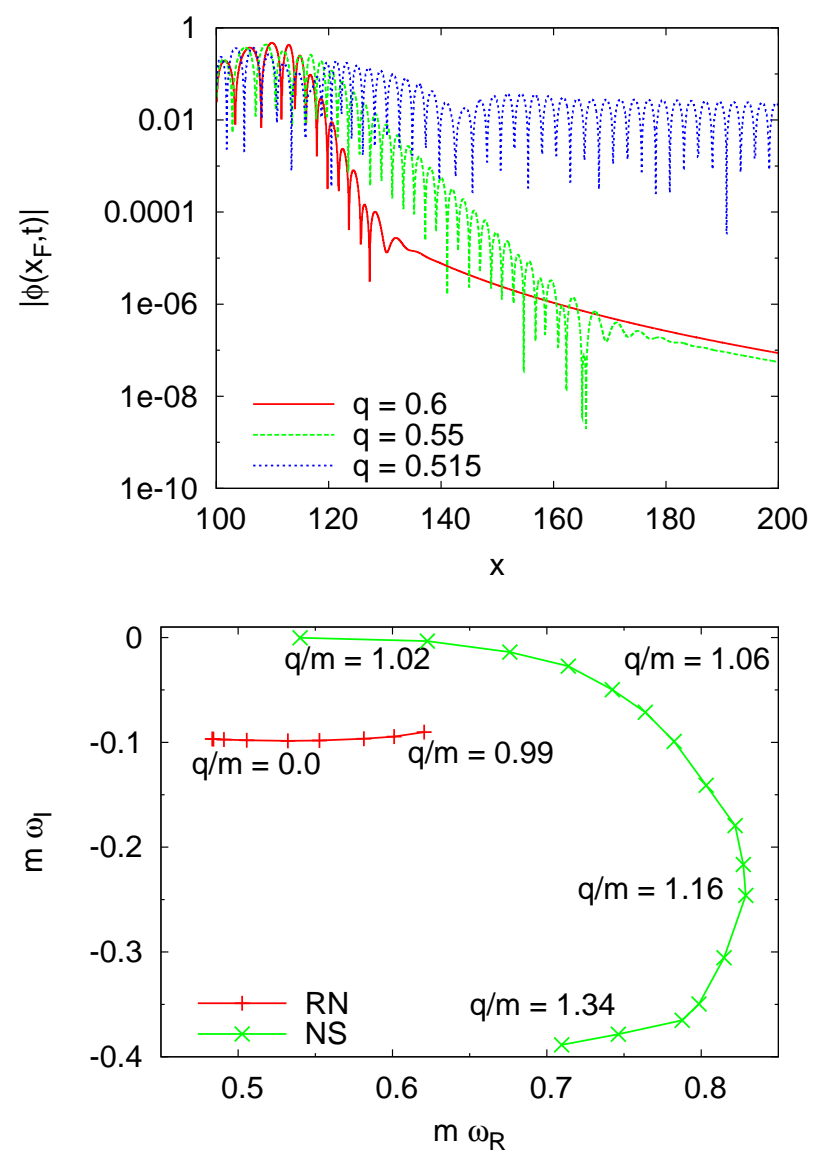

FIG. 6. Above: $\phi\left(x_{F}, t\right)$ with $l=2$ at $x_{F}=100$ for a spacetime with $m=0.5$ and different values of $q>m$. Below: Frequencies of the fundamental mode with $l=2$ in the $\omega_{R} \times \omega_{I}$ plane, parametrized by the $q / m$ ration.

Finally, in fig. 7 we explore how the frequencies of the QNMs change with $l$. As usual in black hole scattering problems, we see that the oscillation frequency $\omega_{R}$ increases with $l$. But the qualitative behaviour of
$\omega_{I}$ changes significantly with $q / m$. In the upper plots $\left(q^{2} / m^{2} \lesssim 9 / 8\right),\left|\omega_{I}\right|$ decreases with $l$, that is, the damping time is longer. In the lower plots $\left(q^{2} / m^{2} \gtrsim 9 / 8\right)$, we have the opposite tendency. This behaviour is connected to the potential $V(r)$ shown in fig. 3. It might be also interesting to mention that in case $q^{2} / m^{2} \gtrsim 9 / 8$, there is a qualitative similarity in the behaviour of the imaginary part of the frequencies as a function of $l$, between the case when $l$ is small and the $l$ large limit.
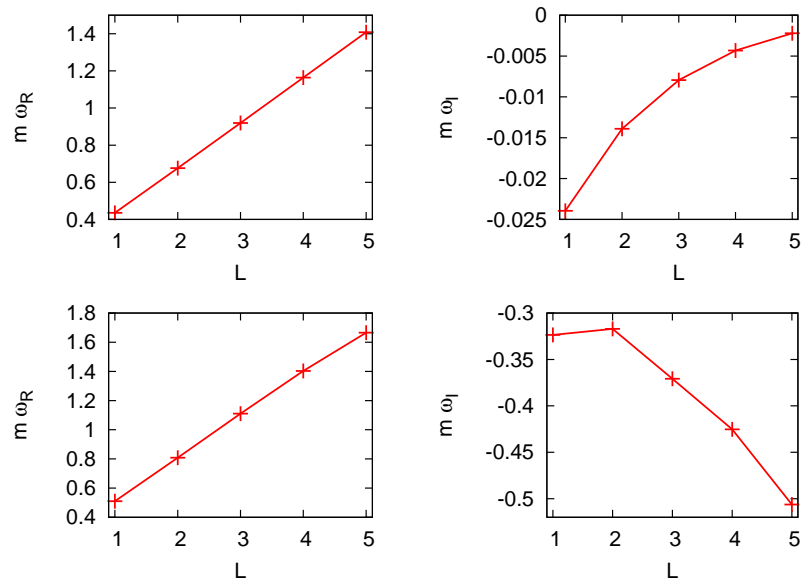

FIG. 7. Above: frequencies of the fundamental mode as a function of $l$ for $m=0.5$ and $q=0.52\left(q^{2} / m^{2}<9 / 8\right)$. Below: same as above, but this time for $m=0.5$ and $q=0.6$ $\left(q^{2} / m^{2}>9 / 8\right)$.

\section{ASYMPTOTICALLY HIGHLY DAMPED MODES FOR THE SCALAR FIELD IN THE R-N NAKED SINGULARITY SPACETIME}

Let us explore the following limit, $l$ will be fixed and $n \rightarrow \infty$, assuming that there exist an infinite number of QNMs. Assume further that $\omega^{2}, \omega_{I}$ diverge as $n \rightarrow$ $\infty$. In such a limit, to have a good understanding of what the QNM boundary conditions are, it is convenient to take an analytic continuation of the solution into the complex $x$ plane and impose the outgoing wave boundary conditions on the (Stokes) line $\operatorname{Im}(\omega x)=0$. This is for almost imaginary asymptotic frequencies $\left(\omega \approx i \omega_{I} \rightarrow\right.$ $-i \infty$ as $n \rightarrow \infty)$ the line $\operatorname{Re}(x)=0$. The key point is to realize that, in the asymptotic case, $\omega^{2} \gg|V(x)|$ holds everywhere apart of a tiny region around 0 , where the potential $V(x)$ can be already approximated by

$$
V(x) \approx-\frac{2}{9 x^{2}}
$$

(There is one subtlety: The potential, and also later the solutions, are multi-valued functions in the complex variable, but after specifying a branch cut together with the branch, this does not cause any problems to our analysis.) 
So let us make the following statement: The solutions of the equation

$$
\frac{d^{2} \phi_{l}(x)}{d x^{2}}+\left(\omega^{2}+\frac{2}{9 x^{2}}\right) \phi_{l}(x)=0
$$

are everywhere good approximations to the solutions of the scalar field equation for the asymptotically highly damped modes. (The region where the approximations $x \approx r^{3}$ and $V(x) \sim 1 / r(x)^{6}$ cease to hold is the region where we can already neglect the potential as a whole with respect to the $\omega^{2}$ term.)

The equation (67) has a general solution given as

$$
\phi_{l}(x)=\sqrt{\omega x}\left[A J_{1 / 6}(\omega x)+B J_{-1 / 6}(\omega x)\right],
$$

where $J_{a}$ is the Bessel function. The solution for which $\psi_{l}(x)$ fulfils the vanishing boundary condition at 0 is given by $B=0$. Our QNM boundary condition says that this solution taken along the Stokes line $\operatorname{Im}(\omega x)=0$, should give purely outgoing radiation for $\omega x \rightarrow \infty$. But in the $\omega x \rightarrow \infty$ limit we know that we can approximate the solution (via Bessel functions approximations in such a limit) as

$$
\begin{aligned}
A \sqrt{\omega x} J_{1 / 6}(\omega x) & \approx A \sqrt{\frac{2}{\pi}} \cos \left(\omega x-\frac{\pi}{3}\right)= \\
& =A \sqrt{\frac{1}{2 \pi}}\left(e^{-i \pi / 3} e^{i \omega x}+e^{i \pi / 3} e^{-i \omega x}\right) .
\end{aligned}
$$

This linear combination is independent on $\omega$ and does not give the purely outgoing radiation $\left(\sim e^{i \omega x}\right)$, which suggests that our QNM boundary conditions cannot be fulfilled for asymptotically highly damped modes.

This can be taken as an evidence that the asymptotically highly damped modes $\left(\omega_{I} \rightarrow-\infty\right)$ do not exist for the R-N naked singularity and this can be taken as a confirmation that the highly damped QNMs could link to the black hole spacetime horizon's properties (non-existence of the horizon leads to non-existence of the modes in the asymptotic limit). One can see also some link to the fact that for the extremal R-N black hole the spacing between the asymptotic frequencies goes to zero as proportional to the surface gravity (this might make a whole infinite tower of arbitrarily highly damped modes eventually "collapse" to a single mode). Moreover, we assume that the non-existence of the highly damped modes can be proven along the same lines also for another naked singularity spacetimes (like negative mass Schwarzschild spacetime etc [9]).

\section{CONCLUSIONS}

In this paper we analysed the problem of the scalar field scattering on a R-N naked singularity background from the point of view of quasi-normal modes. The evolution on the R-N naked singularity is non-unique unless one specifies an additional boundary condition representing a "hair" of the singularity. The quasi-normal modes then carry information about the "hair". We applied a particular boundary condition, that nothing comes out, or in from the singularity and analysed analytically, as well as numerically, the characteristic oscillations of the scalar field perturbations (low damped quasinormal modes). We analysed the eikonal $l \gg 1$ case via the analytical approach confirming the intuition obtained through the massless particle viewpoint, and showed that an approach based on analytical approximations can be useful also for the small $l$ wave mode numbers. For the small $l$-s we calculated the frequencies numerically via the characteristic integration method. We also suggested arguments showing that the asymptotically highly damped modes (limit $l$ fixed and $n \rightarrow \infty$ ) do not exist in case of $\mathrm{R}-\mathrm{N}$ naked singularity. This might confirm the intuition one has about such modes from the black hole physics (and from the presently popular conjectures [11, 12]).

The basic results can be summarized as follows: for the low modes and the large $l$ there is a continuous transition in the low damped QNM modes between the R-N black hole and the R-N naked singularity. However, when the ratio $q^{2} / m^{2}$ becomes larger than approximately $9 / 8$ then the picture becomes significantly different and the low damped modes do not exist for large $l$-s. (This is a very different picture from the $\mathrm{BH}$ based intuition.) For the small $l$ numbers the modes face a discontinuous transition when transiting from the black hole to the naked singularity. Furthermore, the $l$ dependence $\left|\omega_{I}\right|$ (for small l) changes as $q^{2} / m^{2}$ becomes larger than approximately 9/8: $\left|\omega_{I}\right|$ decreases for $q^{2} / m^{2} \lesssim 9 / 8$ and increases for $q^{2} / m^{2} \gtrsim 9 / 8$. It might be interesting to notice that for $q^{2} / m^{2} \gtrsim 9 / 8$ the increase of $\left|\omega_{I}\right|$ as a function of $l$ (for small $l$-s) matches the behaviour of $\left|\omega_{I}\right|$ for large $l$-s. In the case of large $l$-s and $q^{2} / m^{2} \gtrsim 9 / 8$ we have shown that $\left|\omega_{I}\right|$ of the fundamental mode grows at least cubically with $l$ and thus, as we already mentioned, the low damped modes do not exist. For the asymptotically highly damped modes our results seem to suggest that they do not exist, which means that the imaginary parts of the frequencies are bounded.

\section{Acknowledgments}

The authors are grateful to FAPESP, CNPq, and the Max Planck Society for the financial support.

\section{Appendix A: Calculations of some of the important quantities}

In this appendix section we provide a list of some of the quantities (also some of their derivations) that occur in the calculations relevant to the problem analysed in this paper. Write $V(r)$ in a convenient form:

$$
V(r)=\frac{A}{r^{2}}+\frac{B}{r^{3}}+\frac{C}{r^{4}}+\frac{D}{r^{5}}+\frac{E}{r^{6}},
$$


with

$$
\begin{aligned}
A & \equiv l(l+1), \\
B & \equiv 2 m[1-l(l+1)], \\
C & \equiv q^{2} l(l+1)-(2 m)^{2}-2 q^{2}, \\
D & \equiv 6 m q^{2} \\
E & \equiv-2 q^{4} .
\end{aligned}
$$

Now let us calculate the second derivative of the potential with respect to the tortoise coordinate:

$$
\frac{d^{2} V(x)}{d x^{2}}=\frac{d^{2} V(r)}{d r^{2}}\left(\frac{d r}{d x}\right)^{2}+\frac{d V(r)}{d r} \frac{d^{2} r}{d x^{2}}
$$

At $r_{\max }$ the derivative $d V(r) / d r$ is zero, then necessarily

$$
\left.\frac{d^{2} V(x)}{d x^{2}}\right|_{\mid x_{\max }}=\left[\frac{d^{2} V(r)}{d r^{2}}\left(\frac{d r}{d x}\right)^{2}\right]_{\mid x_{\max }}
$$

and since

$$
\frac{d^{2} V(r)}{d r^{2}}=\frac{6 A}{r^{4}}+\frac{12 B}{r^{5}}+\frac{20 C}{r^{6}}+\frac{30 D}{r^{7}}+\frac{42 E}{r^{8}}
$$

we obtain

$$
\begin{aligned}
\frac{d^{2} V(r)}{d x^{2}} & =\left(1-\frac{2 m}{r}+\frac{q^{2}}{r^{2}}\right)^{2} \\
& \times\left(\frac{6 A}{r^{4}}+\frac{12 B}{r^{5}}+\frac{20 C}{r^{6}}+\frac{30 D}{r^{7}}+\frac{42 E}{r^{8}}\right)
\end{aligned}
$$

For the global extremum condition holds:

$$
\frac{d V(r)}{d r}=2 A r_{\max }^{4}+3 B r_{\max }^{3}+4 C r_{\max }^{2}+5 D r_{\max }+6 E=0
$$

This equation can have maximally 4 roots. From (29) it can be easily seen that for $r \rightarrow \infty$ (29) becomes for any $l, q, m$ positive (dominant term is $l(l+1) / r^{2}$ ) and goes to 0 . In case of naked singularity for $r \rightarrow 0$ the potential goes always to $-\infty$ (dominant term is $\left.-2 q^{4} / r^{6}\right)$, in case of black hole for $r \rightarrow r_{+}$it always goes to 0 (dominant term is $f(r))$. This means, together with (A11), that for any $m, q, l$ (black hole or not) there are always either two local maxima and one local minimum, or one local maximum without local minima.

\section{The large $l$ limit}

For $l \gg 1$ the following approximations hold: For large $l$ the maximum of the inner peak can be approximately found analytically and behaves as:

$$
r_{\max } \approx \frac{\sqrt{3}|q|}{\sqrt{l(l+1)}} .
$$

Also holds the following: For $x \ll 1$

$$
x \approx \frac{r^{3}}{3 q^{2}} .
$$

The parameters $\alpha_{1}, V_{1}$ turn in the large $l$ limit to be:

$$
\alpha_{1} \approx \frac{2}{3} \frac{[l(l+1)]^{3 / 2}}{|q|}
$$

$$
\sqrt{V_{1}} \approx \frac{[l(l+1)]^{3 / 2}}{3^{3 / 2}|q|}
$$

This implies also the following results: For $l \rightarrow \infty$

$$
\begin{aligned}
x_{1 \max } \alpha_{1} & \rightarrow \frac{2}{\sqrt{3}}, \\
\frac{\sqrt{V_{1}}}{\alpha_{1}} & \approx \frac{1}{2 \sqrt{3}}, \\
V_{2} & \sim l(l+1), \\
\frac{V_{2}}{V_{1}} & \rightarrow 0 \\
\frac{\sqrt{V_{2}}}{\alpha_{1}} & \rightarrow 0, \\
\frac{\sqrt{V_{2}}}{\alpha_{2}} & \rightarrow \infty, \\
V(x) & \approx-\frac{2}{9 x^{2}} \text { for } \quad x \ll 1 .
\end{aligned}
$$

\section{Appendix B: Some suggestions for the analytical treatment of the scattering for small wave mode numbers}

Write the two linearly independent solutions on the domain $i$ in the form convenient for the logarithmic derivative gluing condition: $C_{i}\left(\Psi_{i 1}+K_{i} \Psi_{i 2}\right)$. If the domain extends to the infinity and $\Psi_{i 1}$ is taken to be the asymptotically incoming wave solution and $\Psi_{i 2}$ the asymptotically outgoing wave solution, then $K_{i}(\omega)$ is the S-matrix (ratio of the coefficients of the outgoing and incoming waves). Anyway, the logarithmic derivative gluing condition at the boundary of the regions $i$ and $i-1$ turns to be: 


$$
K_{i}=\left[\frac{\Psi_{(i-1) 1}^{\prime} \Psi_{i 1}-\Psi_{i 1}^{\prime} \Psi_{(i-1) 1}+K_{i-1}\left(\Psi_{(i-1) 2}^{\prime} \Psi_{i 1}-\Psi_{i 1}^{\prime} \Psi_{(i-1) 2}\right)}{\Psi_{i 2}^{\prime} \Psi_{(i-1) 1}-\Psi_{(i-1) 1}^{\prime} \Psi_{i 2}+K_{i-1}\left(\Psi_{i 2}^{\prime} \Psi_{(i-1) 2}-\Psi_{(i-1) 2}^{\prime} \Psi_{i 2}\right)}\right]_{\left.\right|_{a_{i}}}
$$

This is the condition connecting the $K_{i}$ coefficient with $K_{i-1}$ coefficient through the values of the solutions and their derivatives at the boundary of the regions. On one side consider asymptotically free-wave region, whereas on the other side consider infinite potential barrier-like boundary condition for $\Psi$. In such case one can through equations of the type (B1) connect (after finite number of steps) the S-matrix of the external (asymptotic) region with the boundary condition at the origin (where we can conveniently put $K_{1}$ to be zero). Then one gets the S-matrix expressed through a function of the boundary values (at all the boundaries) of the solutions and their derivatives. This will be algebraically more difficult condition than the one coming just from posing the outgoing wave condition in the external region, as we did in the previous sections (case of large $l$ and $q^{2} / \mathrm{m}^{2}$ larger than approximately $9 / 8$ ). The reason for posing the more complicated condition of the type (B1) is that it might be easier (and also brings more insight) to look for a real energy resonances (by plotting phase change and looking for rapid phase shifts, hence rapid change of the logarithm of the S-matrix), than to try to numerically solve the more simple outgoing wave condition (with the help of appropriate computer software). Such resonances should then 1 to 1 correspond to the low damped quasinormal frequencies. Let us apply these ideas and try to calculate the expressions for the partial S-matrix.

\section{The case for $q^{2} / m^{2} \gtrsim 9 / 8$}

This is the case V1. We are gluing only two regions. The solution on the left side of the boundary $\Psi_{L}$, such that fulfils $\Psi_{L}(0)=0$, is again given as (40). On the right side (the Morse potential side) the solution is given as:

$$
\Psi_{R}(x)=C_{1}\left[\Psi_{R / I}(x)+S\left(\omega, \alpha_{1}, V_{1}\right) \Psi_{R / O}(x)\right]=C_{1} e^{-\frac{z}{2}}\left[e^{-i \omega x} M\left(s_{1}, s_{2}, z\right)+S\left(\omega, \alpha_{1}, V_{1}\right) e^{i \omega x} M\left(s_{1}^{\prime}, s_{2}^{\prime}, z\right)\right] .
$$

By $\Psi_{R / O}(x), \Psi_{R / I}(x)$ we mean asymptotically outgoing and incoming wave solutions in the sense that $\Psi_{R / I}(x \rightarrow$ $\infty) \rightarrow e^{-i \omega x}$ and $\Psi_{R / O}(x \rightarrow \infty) \rightarrow e^{i \omega x}$ exactly. Furthermore $M(., .,$.$) is being the Kummer (confluent) hypergeo-$ metric function and

$$
\begin{aligned}
s_{1} & \equiv \frac{1}{2}-i \frac{\sqrt{V_{1}}}{\alpha_{1}}+\frac{i \omega}{\alpha_{1}} \equiv s_{1}^{\prime}+\frac{2 i \omega}{\alpha_{1}}, \\
s_{2} & \equiv 1+\frac{2 i \omega}{\alpha_{1}} \equiv s_{2}^{\prime}+\frac{4 i \omega}{\alpha_{1}}, \\
z & \equiv \frac{i 2 \sqrt{V_{1}}}{\alpha_{1}} \exp \left(-\alpha_{1}\left(x-x_{1 \text { max }}\right)\right) .
\end{aligned}
$$

Also $S_{l}\left(\omega, \alpha_{1}, V_{1}\right)$ is the S-matrix. The logarithmic derivatives gluing condition at $a_{1}=x_{1 \max }-\frac{1}{\alpha_{1}} \ln (2)$ is then

$$
f\left(a_{1}\right)\left[\frac{1}{a_{1}}+\frac{\beta_{1} e^{\beta_{1} a_{1}}-\beta_{2} e^{\beta_{2} a_{1}}}{e^{\beta_{1} a_{1}}-e^{\beta_{2} a_{1}}}\right]=\frac{\alpha_{1} z\left(a_{1}\right)}{2}+i \omega+\frac{s_{1}^{\prime}}{s_{2}^{\prime}} \frac{M\left(s_{1}^{\prime}+1, s_{2}^{\prime}+1, z\left(a_{1}\right)\right)}{M\left(s_{1}^{\prime}, s_{2}^{\prime}, z\left(a_{1}\right)\right)} .
$$

Considering that the following holds:

$$
z\left(a_{1}\right)=\frac{i 4 \sqrt{V_{1}}}{\alpha_{1}}
$$

one can rewrite (Bי) into:

$$
f\left(a_{1}\right)\left[\frac{1}{a_{1}}+\frac{\beta_{1} e^{\beta_{1} a_{1}}-\beta_{2} e^{\beta_{2} a_{1}}}{e^{\beta_{1} a_{1}}-e^{\beta_{2} a_{1}}}\right]=i 2 \sqrt{V_{1}}+i \omega+\frac{s_{1}^{\prime}}{s_{2}^{\prime}} \frac{M\left(s_{1}^{\prime}+1, s_{2}^{\prime}+1, \frac{i 4 \sqrt{V_{1}}}{\alpha_{1}}\right)}{M\left(s_{1}^{\prime}, s_{2}^{\prime}, \frac{i 4 \sqrt{V_{1}}}{\alpha_{1}}\right)} .
$$

The partial S-matrix can be obtained from (B1) $\left(K_{1}=0\right)$ as:

$$
S_{l}\left(\omega, V_{1}, \alpha_{1}\right)=-\frac{\Psi_{R / I}}{\Psi_{R / O}} \frac{\frac{\Psi_{L}^{\prime}}{\Psi_{L}}-\frac{\Psi_{R / I}^{\prime}}{\Psi_{R / I}^{\prime}}-\frac{\Psi_{R / O}^{\prime}}{\Psi_{R / O}}}{\Psi_{L}}
$$

Since for real $\omega$ holds that $\Psi_{R / O}=\Psi_{R / I}^{*}$ and $\Psi_{L}$ is a real valued function, one can immediately observe that 
(for $\omega \in \mathbb{R}) S_{l}^{*}(\omega)=S_{l}^{-1}(\omega)$, hence partial S-matrix is unitary.

From (B9) we can conclude the following

$$
\begin{aligned}
& S_{l}\left(\omega, V_{1}, \alpha_{1}\right)=-e^{-2 i \omega a_{1}} \frac{M\left(s_{1}, s_{2}, \frac{i 4 \sqrt{V_{1}}}{\alpha_{1}}\right)}{M\left(s_{1}^{\prime}, s_{2}^{\prime}, \frac{i 4 \sqrt{V_{1}}}{\alpha_{1}}\right)} \\
& \times \frac{f\left(a_{1}\right)\left[\frac{1}{a_{1}}+\frac{\beta_{1} e^{\beta_{1} a_{1}}-\beta_{2} e^{\beta_{2} a_{1}}}{e^{\beta_{1} a_{1}}-e^{\beta^{2} a_{1}}}\right]-\left[i 2 \sqrt{V_{1}}-i \omega+\frac{s_{1}}{s_{2}} \frac{M\left(s_{1}+1, s_{2}+1, \frac{i 4 \sqrt{V_{1}}}{\alpha_{1}}\right)}{M\left(s_{1}, s_{2}, \frac{i 4 \sqrt{V_{1}}}{\alpha_{1}}\right)}\right]}{f\left(a_{1}\right)\left[\frac{1}{a_{1}}+\frac{\beta_{1} e^{\beta_{1} a_{1}}-\beta_{2} e^{\beta_{2} a_{1}}}{e^{\beta_{1} a_{1}}-e^{\beta_{2} a_{1}}}\right]-\left[i 2 \sqrt{V_{1}}+i \omega+\frac{s_{1}^{\prime}}{s_{2}^{\prime}} \frac{M\left(s_{1}^{\prime}+1, s_{2}^{\prime}+1, \frac{i 4 \sqrt{V_{1}}}{\alpha_{1}}\right)}{M\left(s_{1}^{\prime}, s_{2}^{\prime}, \frac{i 4 \sqrt{V_{1}}}{\alpha_{1}}\right)}\right]}
\end{aligned}
$$

2. The two peak case, $q^{2} / m^{2} \lesssim 9 / 8$

The two peak case is a more complicated problem. Anyway, one can write the logarithmic derivative gluing condition as

$$
\begin{aligned}
\frac{\alpha_{1} z\left(a_{2}\right)}{2} & -i \omega \frac{S_{1}\left(\omega, \alpha_{1}, V_{0}\right) M\left\{s_{1}, s_{2}, z\left(a_{2}\right)\right\}+e^{i 2 \omega a_{2}} M\left\{s_{1}^{\prime}, s_{2}^{\prime}, z\left(a_{2}\right)\right\}}{S_{1}\left(\omega, \alpha_{1}, V_{0}\right) M\left\{s_{1}, s_{2}, z\left(a_{2}\right)\right\}-e^{i 2 \omega a_{2}} M\left\{s_{1}^{\prime}, s_{2}^{\prime}, z\left(a_{2}\right)\right\}} \\
& +\frac{S_{1}\left(\omega, \alpha_{1}, V_{0}\right) M\left\{s_{1}+1, s_{2}+1, z\left(a_{2}\right)\right\} \frac{s_{1}}{s_{2}}-e^{i 2 \omega a_{2}} M\left\{s_{1}^{\prime}+1, s_{2}^{\prime}+1, z\left(a_{2}\right)\right\} \frac{s_{1}^{\prime}}{s_{2}^{\prime}}}{S_{1}\left(\omega, \alpha_{1}, V_{0}\right) M\left\{s_{1}, s_{2}, z\left(a_{2}\right)\right\}-e^{i 2 \omega a_{2}} M\left\{s_{1}^{\prime}, s_{2}^{\prime}, z\left(a_{2}\right)\right\}} \\
& =i \omega \frac{F_{21}\left\{g_{1}, g_{2}, g_{3}-1,\left[1+\exp \left(2 \alpha_{2}\left(a_{2}-x_{2 \max }\right)\right)\right]^{-1}\right\}}{F_{21}\left\{g_{1}, g_{2}, g_{3},\left[1+\exp \left(2 \alpha_{2}\left(a_{2}-x_{2 \max }\right)\right)\right]^{-1}\right\}} .
\end{aligned}
$$

Here $S_{1}$ is the S-matrix from the case V1 and is given by the formula (B10). The S-matrix related to the case V3 (call it $S_{2}$ ) is related to $S_{1}$ through the condition (B1). In the gluing formula (B1) $S_{2}$ stands for $K_{i}$ and $S_{1}$ for $K_{i-1}$. Also $\Psi_{i 1,2}$ are the solutions of the Poeschl-Teller case

$$
\Psi_{i 1}(x)=e^{i \omega x} F_{21}\left[g_{1}, g_{2}, g_{3},\left[1+\exp \left(2 \alpha_{2}\left(x-x_{2 \max }\right)\right)\right]^{-1}\right],
$$

and

$$
\Psi_{i 2}(x)=e^{-i \omega x} F_{21}\left[g_{1}, g_{2}, g_{3},\left[1+\exp \left(-2 \alpha_{2}\left(x-x_{2 \max }\right)\right)\right]^{-1}\right]
$$

$\Psi_{(i-1) 1,2}$ are the Morse potential solutions given as (B2). We see that if there is a regime in which the modes do not feel the smaller peak, then they are given by the poles of $S_{1}$ and must be in the same time poles of $S_{2}$. The formula for $S_{2}$ in this case (for frequencies that represent poles of $S_{1}$ ) simplifies to

$$
S_{2}=\left[\frac{\left(\Psi_{(i-1) 2}^{\prime} \Psi_{i 1}-\Psi_{i 1}^{\prime} \Psi_{(i-1) 2}\right)}{\left(\Psi_{i 2}^{\prime} \Psi_{(i-1) 2}-\Psi_{(i-1) 2}^{\prime} \Psi_{i 2}\right)}\right]_{\Psi_{\omega_{2}}} .
$$

There is a possibility that for the case of the low modes in the two peak model one might be able to neglect the infinite depth of the valley between the infinite barrier and the Morse peak. In such case one can take a simpler model where the Morse potential directly follows after the infinite barrier. The $S_{1}$ term appearing in the formula (B11) will be in such case given by a much more simple expression:

$$
S_{1}\left(\omega, \alpha_{1}, V_{0}\right)=\frac{M\left\{s_{1}, s_{2}, z(0)\right\}}{M\left\{s_{1}^{\prime}, s_{2}^{\prime}, z(0)\right\}} .
$$

[1] M. Richartz and A. Saa, "Challenging the weak cosmic censorship conjecture with charged quantum particles", 
[2] G. Dotti, R. Gleiser and J. Pullin, "Instability of charged and rotating naked singularities", Phys.Lett.B 644:289293, 2007, arXiv:gr-qc/0607052

[3] R.M. Wald, "Dynamics in nonglobally hyperbolic, static spacetimes", J.Math.Phys 21, 2802, 1980,

[4] A. Ishibashi and R.M. Wald, "Dynamics in NonGlobally-Hyperbolic Static Spacetimes II: General Analysis of Prescriptions for Dynamics", Class.Quant.Grav. 20, 2003, 3815-3826, arXiv:gr-qc/0305012,

[5] V.D. Sandberg, "Light scattering properties of naked singularities", Phys.Rev.D 12 2226, (1975)

[6] G. Gibbons, "Quantized fields propagating in plane wave spacetimes", Comm.Math.Phys 45 191, (1975)

[7] G. T. Horowitz and D. Marolf, "Quantum Probes of Spacetime Singularities", Phys.Rev.D 52 (1995) 56705675, arXiv:gr-qc/9504028

[8] J.P.M. Pitelli and P.S. Letelier, "Quantum Singularities in Static Spacetimes", Int.J.Mod.Phys.D 20:729-743, (2011), arXiv:gr-qc/1010.3052

[9] A. Ishibashi and A. Hosoya, "Who's afraid of naked singularities?", PhysRevD. 60 104028, 1999, arXiv:gr-qc/9907009

[10] M. Martelini, C. Reina and A. Treves, "Klein-Gordon field in a naked singularity background", PhysRevD 17 10,1978

[11] S. Hod, "Bohr's correspondence principle and the area spectrum of the quantum black holes", Phys.Rev.Lett. 81, 4293 (1998), arXiv:gr-qc/9812002

[12] M. Maggiore, "The physical interpretation of the spectrum of black hole quasi-normal modes", Phys.Rev.Lett. 100, 141301 (2008), arXiv:gr-qc/0711.3145

[13] J. Skakala, "Quasi-normal modes, area spectra and multi-horizon spacetimes", JHEP 1206:094, 2012,
(2012), arXiv:gr-qc/1204.3566

[14] H.P. Nollert and B.G. Schmidt, "Quasinormal modes of Schwarzschild black holes: Defined and calculated vie Laplace transform", Phys.Rev.D 45 2617, 1992

[15] E.W. Leaver, "Spectral decomposition of the perturbation response of the Schwarzschild geometry", Phys.RevD 34 384, 1986

[16] H.P. Nollert, "Quasinormal modes: the characteristic "sound" of black holes and neutron starts", Class.Quant.Grav 16 (1999) R159

[17] V. Cardoso, A.S. Miranda, E. Berti, H. Witek and V.T. Zanchin, "Geodesic stability, Lyapunov exponents and quasinormal modes", Phys.Rev.D 79 (2009) 064016, arXiv:gr-qc/0812.1806

[18] S. Iyer and C. Will, "Black hole normal modes: A WKB approach.1.Foundations and application of a higher order WKB analysis of potential barrier scattering", PhysRevD 39 (1987) 3621

[19] V. Ferrari and B. Mashhoon "New approach to the quasinormal modes of a black hole", Phys.RevD 30 (1984) 295

[20] G. Rawitscher, C. Merow, M. Nguyen and I. Simbotin, "Resonances and quantum scattering for the Morse potential as a barrier", Am.J.Phys 70 (2002) 935-944, arXiv:nucl-th/0111027

[21] P. Boonserm and M. Visser, "Quasi-normal frequencies: key analytic results", JHEP 1103 073, 2011, arXiv:math-ph/1005.4483

[22] C. Chirenti and A. Saa,"Nonstationary regime for quasinormal modes of the charged Vaidya metric", Phys.Rev. D84 (2011) 064006, arXiv:1105.1681 [gr-qc] 